\title{
Strain Effect on Magneto-optical and Magnetic Properties of Film System Based on Fe and Pt
}

\author{
I.V. Cheshko, Z.M. Makukha, L.V. Odnodvorets, M.O. Shumakova, D.V. Velykodnyi, I.Yu.Protsenko* \\ Sumy State University, Sumy, 40007, Ukraine \\ *Corresponding Author: protsenko@aph.sumdu.edu.ua
}

Copyright $(2013$ Horizon Research Publishing All rights reserved.

\begin{abstract}
In the work the strain effect in magneto-optical (magneto-optical Kerr effect - MOKE) and magnetic properties of thin films and multilayers based on $\mathrm{Fe}$ and $\mathrm{Pt}$ in unstrained and strained states was studied. The proposed ratio for strain coefficient of MOKE us quantitative characteric this effect. By measuring the Kerr angle for different values of strain and geometry measurements, it is concluded the influence of strain effects on magnetic characteristics of two-layer films and multilayers.
\end{abstract}

Keywords Strain effect, Kerr angle, Strain Coefficient of MOKE

\section{Introduction}

Effectiveness of nanodimentional thin film materials as sensitive elements of temperature, pressure, strain, magnetic field and others sensors possible to develop a concept of multi-sensors, including sensors, strain and magnetic field, the principle of the latter may be based on magneto-strain effect.

The principle of operation of these sensors is based on the strain effects of electric resistance $(R)$ film (see, for example, [1-3]) or giant magnetoresistance (GMR) [4, 5]. As a kind of strain effect can be seen magneto-strain effect when the strain coefficient measured in the presence of an external magnetic field $(B)$. Phenomenological theory of this effect in single-films and multilayers proposed in works $[6,7]$ and for now gained some experimental data. In particular, in [8] studied the features of the influence of the magnetic field by a strain coefficient in bulk amorphous alloys based on Fe. In this case, as in the case of diamond films [9], the longitudinal and transverse strain coefficients decreased under the influence of an external magnetic field, that is connected with a change of Young's modulus under the influence of a magnetic field in metallic materials [8], and in the case of diamond films - changing the width of the energy gap [9].

Should be noted that [6 - 9] publications about magneto-strain effect have bailed out.

The quantitative characteristic of strain effect is known is the of longitudinal $\left(\gamma_{l}^{R}\right)$ and transverse $\left(\gamma_{t}^{R}\right)$ strain coefficient, defined by the relation:

$$
\gamma_{l, t}^{R}=\frac{d \ln R}{d \ln \varepsilon_{l, t}}=\frac{1}{R} \cdot \frac{\Delta R}{\Delta \varepsilon_{l, t}} .
$$

Similar strain coefficients for GMR and magneto-strain effect can be defined as follows:

$$
\gamma_{l, t}^{G M R}=\frac{1}{G M R} \cdot \frac{\Delta G M R}{\Delta \varepsilon_{l, t}}
$$

and

$$
\beta_{\gamma_{l, t} B}=\frac{1}{\gamma_{l, t}} \cdot\left(\frac{\Delta \gamma_{l, t}}{\Delta B}\right)_{\varepsilon_{l, t}} .
$$

One of the tasks of our research is to try to extend the range of strain effects that can be used to create strain sensors. It deals with the strain effect in magnetooptical properties (magnetooptical Kerr effect - MOKE) of the thin films and multilayers $\mathrm{Fe} / \mathrm{Pt} / \mathrm{S}$ and $[\mathrm{Fe} / \mathrm{Pt}]_{8} / \mathrm{S} \quad(\mathrm{S}-$ substrate $\left.)\right)$ in unstrained or strained states. In case of MOKE we first proposed the idea and carried out preliminary research strain effect in MOKE. In this regard naturally in the literature no article nor in terms of ideas, nor in terms of experimental results on this effect.

The basis of the effect idea of such considerations. Since different industrial products, apparatus and devices can operate at the a simultaneous strain and magnetic field, then the elastic strain sensors can be used in which performance parameters are the coefficients $\gamma_{l, t}^{R}$ and $\gamma_{l, t}^{G M R}$. However, the transition to the region of plastic strain coefficient $\gamma_{l, t}^{R} \cong 2$ (for more details see [3]), and the behavior of GMR sensing element generally known (for example, plastic strain it might just ruined). At the same time, general considerations suggest that an increase in the value of the strain effect MOKE will be enhanced by increasing the size 
of the magnetic domains.

In accordance with the above, the aim of our work was to study the effect of MOKE and magnetic properties of two-layer films (as a fragments multilayers) and multilayers based on Fe and Pt films, although the choice of film is not of a fundamental nature. Similar studies will be continued by us as an example to magnetic film systems based on $\mathrm{Co}$ and $\mathrm{Cu}$, $\mathrm{Ag}$ or $\mathrm{Au}$ in which granular state is stabilized.

The quantitative characteristic effect seen by analogy with the equations (1) and (2) such a strain coefficient:

$$
\gamma_{l, t}^{M O K E}=\frac{1}{\Theta_{0}} \cdot \frac{\Delta \Theta}{\Delta \varepsilon_{l, t}}
$$

where $\Theta_{0}-$ angle of the rotation of polarization plane at the $\varepsilon=0, \Delta \Theta=\Theta_{\varepsilon_{l, t}}-\Theta_{0} \quad\left({ }^{\varphi} \varepsilon_{l, t}-\right.$ angle of rotation the polarization plane with $\left.\varepsilon_{l, t} \neq 0\right)$.

\section{Experimental Methods and Techniques}

Film samples based on $\mathrm{Fe}$ and $\mathrm{Pt}$ were prepared by condensation of layered crucibles in ultra-high vacuum of $10^{-6} \mathrm{~Pa}$ at substrate of polystyrene (the study of strain effects in MOKE) and single crystal silicon with a natural layer of $\mathrm{SiO}_{2}$ (measurement of magnetic properties). In this case the condensation layers took place at the $T_{s}=350 \mathrm{~K}$, which contributed to a large extent, preservation of identity of the individual layers and the formation of disordered solid solutions, most likely took place at the interface of layers.

The two-layer systems $\mathrm{Fe}(32) / \mathrm{Pt}\left(\mathrm{d}_{\mathrm{Pt}}\right) / \mathrm{S}$ and multilayers $\left[\mathrm{Fe}(3) / \mathrm{Pt}\left(\mathrm{d}_{\mathrm{Pt}}\right)\right]_{8} / \mathrm{S}$, where the thickness of the layers $\mathrm{d}_{\mathrm{Pt}} \cong 3,5$ and $10 \mathrm{~nm}$ were investigated.

For study of the magnetic properties ( $B$ varied from $(-140)$ to $140 \mathrm{mT}$ ) we used magnetic meridian optic Kerr effect. In our case, the vector magnetic field was directed parallel to the sample surface and always lying in the plane of polarization of the incident wave. When changing the external magnetic field intensity of the reflected beam is varied, and there was a rotation of the polarization plane of the world resulting in linearly polarized light after reflection becomes elliptically polarized. Hysteresis loop changed its shape by changing the thickness of the film material and its strain. The shape of the hysteresis loop is determined coercivity $\left(B_{c}\right)$, residual magnetization $\left(B_{r}\right)$ and saturation magnetization $\left(B_{s}\right)$.

Note that in this case the action of an external magnetic field is auxiliary and is magnetized ferromagnetic samples.

Experimental studies MOKE performed in an external magnetic field during strain to $10 \%$, allowing to carry out research both in elastic (up $0.5 \%$ ) and plastic (in the range of $0.5-10 \%)$ strain. The strain was carried out when its direction and the direction of the magnetic field lines are parallel (we denote the index $\langle l\rangle$ ) or perpendicular (index $\langle t »)$, respectively. Note that this indexing is to a large extent arbitrary and has the same meaning as in the case of strain effect (equation (1)). It deals with a variety of measurement geometries MOKE.

\section{Experimental Results}

The methodology of our work is that by measuring the angle of the rotation plane of polarization $(\Theta)$ (Kerr angle) for different values of strain and geometry measurements, it can be concluded regarding the influence of strain effects on magnetic properties of two-layer films and multilayers. Figure 1 shows the dependence of the relative change of the Kerr angle in a wide of strain range of the sample. Note that this interval is almost completely corresponds to the plasticity as a transition from elastic to plastic strain, according to our data, the thin film solid solutions based on Fe and Pt occurs at $\varepsilon_{\text {tr }}=0.4 \%$.

By analogy with the Faraday effect can be written (see, for example, [10]) the following relationship:

$$
\frac{\Theta_{\varepsilon}}{\Theta_{0}}=\frac{(h-\Delta h)}{h} \cdot \frac{(\partial n / \partial \omega) \varepsilon}{(\partial n / \partial \omega)_{0}} \sim \frac{(\partial n / \partial \omega) \varepsilon}{(\partial n / \partial \omega)_{0}},
$$

where $h$ and $\Delta h-$ traversed path of electromagnetic waves in the region of the magnetic field and its variation, respectively; $n$ - refractive index.

Since the first factor $(h-\Delta h) / h$ varies within $1-0.9$, the dependence $\Theta_{\varepsilon_{l}}$ from $\varepsilon_{l}$ can be explained by amplification dispersion relations $n(\omega)$ in the elongation of magnetic domains.

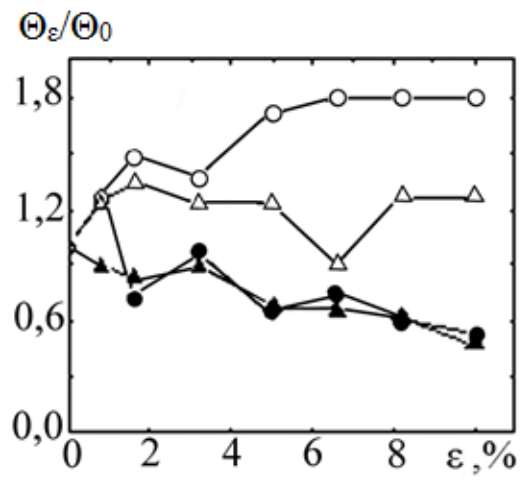

Figure 1. Dependence of the rotation plane of polarization in the longitudinal $(\circ, \Delta)$ and transverse $(\bullet, \mathbf{\Delta})$ geometry measurements for films $\mathrm{Fe}(32) / \operatorname{Pt}(3) / \mathrm{S}(\circ, \bullet)$ and $[\mathrm{Fe}(3) / \operatorname{Pt}(3)] 8 / \mathrm{S}(\Delta, \boldsymbol{\Delta})$. Here and below - the thickness in $\mathrm{nm}$

Since the magneto-optical effect in ferromagnets is determined by the effective internal fields caused by the exchange and spin-orbit interaction, then the strain at the microscopic level, the processes that cause change in the effective field and the resulting birefringence for ferromagnetic.

The peculiarity of these changes is characterized by the 
ratio $\frac{(\partial n / \partial \omega) \varepsilon}{(\partial n / \partial \omega)_{0}}$, which has a value $\sim 1$

Based of dependencies $\Theta_{\varepsilon} / \Theta_{0}$ versus $\varepsilon$ (a typical example is shown in Figure 1) and equation (3), which can be represented as follows:

$$
\gamma_{l, t}^{M O K E}=\frac{1}{\Delta \varepsilon_{l, t}} \cdot\left(\frac{\Theta_{\varepsilon}}{\Theta_{0}}-1\right),
$$

coefficients $\gamma_{l}^{M O K E}$ were calculated, the value of which increases with increasing common concentration of $\mathrm{Fe}$ atoms. At the same time $\gamma_{t}^{M O K E}$ does not depend on the value of $\varepsilon_{t}$ the common concentration of $\mathrm{Fe}$ atoms.

Coefficient $\gamma_{l}^{M O K E}$ varies in the range from 8 units (for $\mathrm{Fe}(32) / \mathrm{Pt}(3) / \mathrm{S}$ ) to 2.5 units (for $\mathrm{Fe}(32) / \mathrm{Pt}(10) / \mathrm{S}$ ) and from 3 units (for multilayers $[\mathrm{Fe}(3) / \mathrm{Pt}(3)]_{8} / \mathrm{S}$ ) to 1.2 units (for multilayers $\left.[\mathrm{Fe}(3) / \mathrm{Pt}(10)]_{8} / \mathrm{S}\right)$. Coefficient $\gamma_{t}^{M O K E} \cong-(4.0$ - 4.5) units for all investigated two-layer films and multilayers. Such a change between $\gamma_{l}^{M O K E}$ and $\gamma_{t}^{M O K E}$, as hysteretic dependences, presented in Figure 2, we explain the different character of the interaction electromagnetic waves with electron gas at the $l$-or $t$-geometry measurement of MOKE.

Note that the value $\gamma_{l, t}^{M O K E}$ play the same role in the strain effects of MOKE as $\gamma_{l, t}^{R}$ and $\gamma_{l, t}^{G M R}$ on the strain effect of resistance and GMR, that can be used as a working option strain sensors, which are based on the effect of MOKE.

Figure 2 shows an example dependency MOKE obtained at different values of strain, the magnitude of the external field.

We obtained values of the Kerr angle can be qualitatively compared with the data of work [11] for ordered and desoreded phase $\mathrm{Fe}-\mathrm{Pt}$ alloys film. For phase $\mathrm{Fe}_{\mathrm{x}} \mathrm{Pt}_{1-\mathrm{x}}$ alloys $(x=27-53$ at.\% $)$ the angle Kerr, depending on the heat treatment conditions, changes in the range $0-35^{\prime}$. In the case of single-layer films Fe value $\Theta$ varies from 17 to 35 ', which to some extent consistent with our data for the two-layer system and multilayer (Figure 2) based on $\mathrm{Fe}$ and Pt. These data show that in this case there is a partial mixing of atoms of Fe and Pt. Obviously, such mixing will occur at the interfaces.

Processing of experimental dependencies, such as those presented on Figure 2, provides a dependence of magnetic characteristics $B_{c}, B_{r}$ and $B_{s}$ on the thickness of the individual layers or strain value. On Figure 3 presents examples of these relationships for $B_{c}$. Their distinctive feature is that the $\mathrm{t}$-geometry measurement MOKE there is much less value $B_{c}$ compared to $l$-geometry measurement, although in both cases the value $B_{c}$ increases. Unlike $B_{c}$ value $B_{r}$ does not change with increasing strain and the value of $B_{s}$ decreases monotonically.

A significant reduction $B_{c}$ takes place with increasing thickness of the paramagnetic layer of Pt in two-layer film $\mathrm{Fe}\left(\mathrm{d}_{\mathrm{Fe}}=\right.$ const $) / \mathrm{Pt}(\mathrm{x}) / \mathrm{S}$ or the number $B_{c}$, in double layer film $\mathrm{Fe}(32) / \mathrm{Pt}(3) / \mathrm{S}$ the value $B_{c}$ decreases from $33(\mathrm{x} \approx 3 \mathrm{~nm})$ to $26(\mathrm{x} \approx 15 \mathrm{~nm}) \mathrm{mT}$, and in the case multilayer $[\mathrm{Fe}(3) / \mathrm{Pt}(3)]_{\mathrm{n}} /$ $\mathrm{S}$ the value $B_{c}$ decreases from $38(\mathrm{n}=2)$ to $30(\mathrm{n}=8) \mathrm{mT}$.
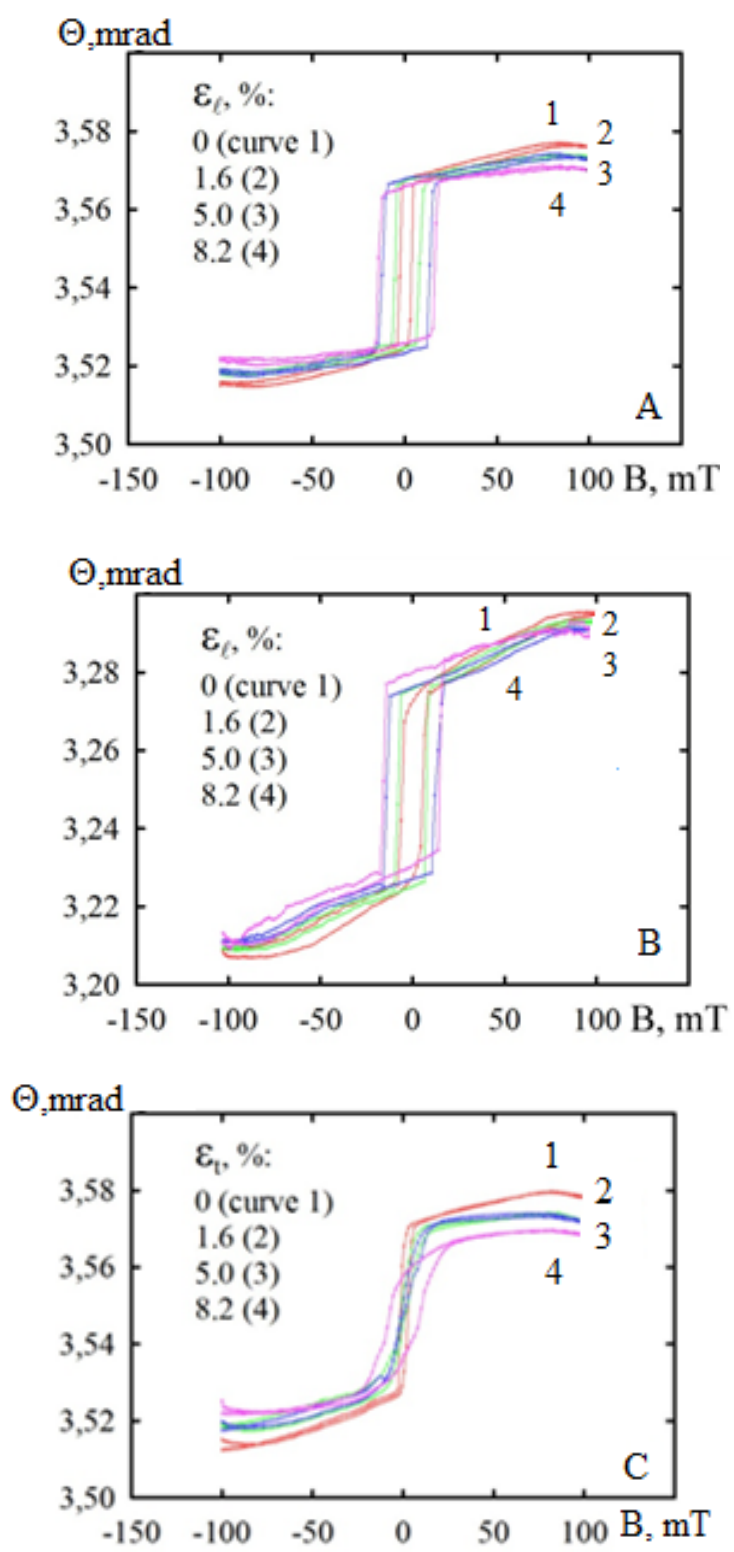

Figure 2. Hysteresis depending MOKE for films $\mathrm{Fe}(32) / \mathrm{Pt}(3) / \mathrm{S}(\mathrm{A}, \mathrm{C})$ and $[\mathrm{Fe}(3) / \mathrm{Pt}(3)]_{8} / \mathrm{S}(\mathrm{B})$ at the $l-(\mathrm{A}, \mathrm{B})$ and $t-(\mathrm{C})$ measurement geometries MOKE

Our results are presented in Figure 3, a consistent quality that you would expect, because the types of samples are very different. From this point of view is more correct comparison of the results of work [15], in which the data for the $B_{c}$ multilayer $[\mathrm{Pt}(3.2) / \mathrm{Fe}(4.8)]_{10}$ at the condensation in the interval $T_{s}=620-770 \mathrm{~K}$. The authors [15] observed an increase in $B_{c}$ from 130 to $950 \mathrm{mT}$ with increasing $T_{s}$ from 620 to $770 \mathrm{~K}$. Similar results were also obtained by the authors [16] for multilayer $[\mathrm{Fe}(1.5) / \mathrm{Pt}(1.5)]_{13}$, which coercivity changed at the annealing $B_{c}=0\left(T_{s}=570 \mathrm{~K}\right)$ to 1,3 
$\mathrm{T}\left(T_{s}=820 \mathrm{~K}\right)$. Since our data for the $B_{c}$ obtained at the $T_{s}=$ $350 \mathrm{~K}$, we can talk about satisfactory agreement our and authors of works $[15,16]$ results.
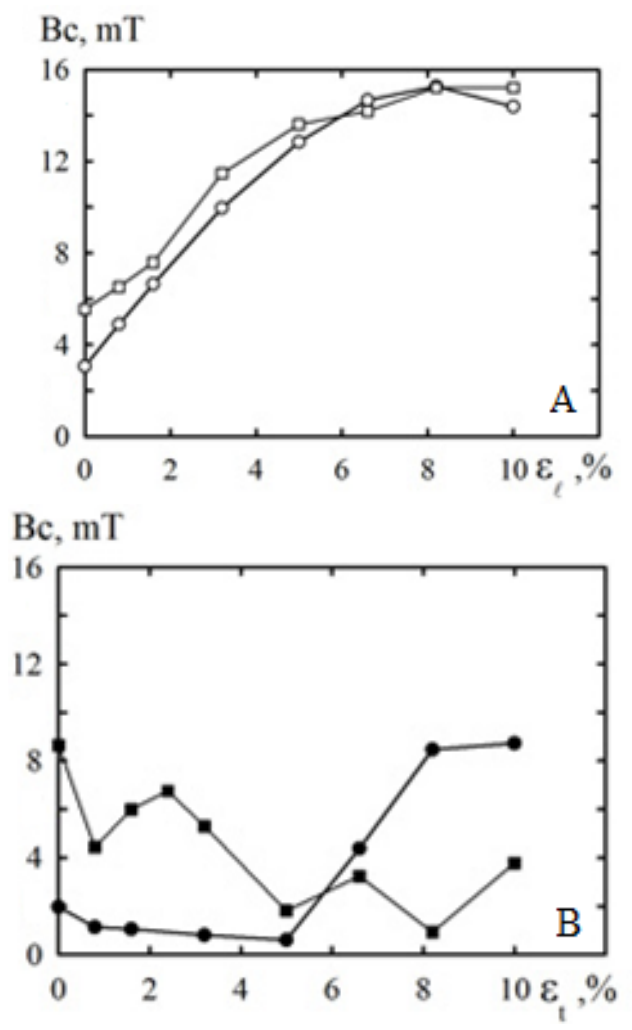

Figure 3. The typical dependence $B_{c}$ versus $\varepsilon_{l}$. ०, • - double-layer films $\mathrm{Fe}(32) / \mathrm{Pt}(3) / \mathrm{S}$ and $\Delta, \boldsymbol{\Delta}$ - multilayer $[\mathrm{Fe}(3) / \mathrm{Pt}(3)]_{8} / \mathrm{S}$ in $l$ - (A) and $t$ - (B) measurement geometries

\section{Conclusion}

The results can be qualitatively explained if we proceed from the assumption that the strain of the sample causes resizing domains and effective magnetic fields. Along with some influence of strain effect on magnetic properties has, let's call it so, a concentration effect, which is caused by the formation and partial ordering of the solid solution based on atoms of $\mathrm{Fe}$ and $\mathrm{Pt}$. We studied the new strain effect of MOKE with strain coefficient $\gamma_{l, t}^{M O K E}$ of the same order of magnitude as the range $\gamma_{l, t}^{R}$ and $\gamma_{l, t}^{G M R}$ can be used to create strain sensors magnetostriction materials.

\section{Acknowledgements}

The authors express gratitude to Prof. Nepijko S.A. (Johannes Gutenberg-Universität Mainz, Germany) for discussion of research results, and one of them D.V.Velykodnyi expresses gratitude to the DAAD Fund (Germany) for financial support for the study of magnetooptical and magnetic properties of film materials, including and these studies.

\section{REFERENCES}

[1] S.I. Protsenko, D.V. Velykodnyi, V.A. Kheraj, M.S. Desai, C.J. Panchal, I.Yu. Protsenko, Electrophysical properties of $\mathrm{Cu} / \mathrm{Cr}$ and $\mathrm{Fe} / \mathrm{Cr}$ films systems within elastic and plastic deformation range, Journal of Materials Science, 2009, Vol. 44, №18, 4905-4910.

[2] L.V.Odnodvorets, S.I.Protsenko, A.M.Chornous, I.Yu.Protsenko, Effect of tensosensibility in metal film materials, Uspehi Fiziki Metallov., 2007, Vol. 8, №2, 109156 (Ukr.).

[3] I.Yu. Protsenko, L.V.Odnodvorets, K.V. Tyschenko, M.O. Shumakova, Features strain properties thin-films: anomalous small of strain coefficient, Journal of Mechanical Engineering and Technology, 2013, Vol.1, Iss.1, 35 - 40.

[4] B. Anwarzai, V. Ac, S. Luby, E. Majkova, R. Sendera, Pseudo spin-valve on plastic substrate as sensing elements of mechanical strain, Vacuum, 2010, Vol.84, $108-110$.

[5] S. Luby, B. Anwarzai, V. Aĉ, E. Majkova, R. Senderak, Pseudo spin-valve with different spacer thickness as sensing elements of mechanical strain, Vacuum, 2012, Vol.86, 718 720 .

[6] S.I. Protsenko, Magneto-deformation effect in thin films, Journal of Nano- and Electronic Physiscs, 2009, Vol.1, №2, 5-7.

[7] Z.M. Makukha, S.I. Protsenko, L.V. Odnodvorets, I.Yu. Protsenko, Magneto -Strain Effect in Double-Layer Film Systems, Journal of Nano- and Electronic Physiscs, 2012, Vol. 4, №2, 02043 - 02045.

[8] M.P. Semen'ko, M.I. Zakharenko, Yu.A. Kunyts'kyi, A.P. Shpak, Strain resistive of amorphous ferromagnets, Uspehi Fiziki Metallov, 2009, Vol.10, №4, 331 - 388 (Ukr.).

[9] W.L. Wang, K.J. Liao, C.G. Hu, S.X. Wang, C.Y. Kong, H.Y. Liao, Study on piezoresistive effect of diamond films under magnetic field, Sensors and Actuatuators A, 2003, Vol. $108,55-58$.

[10] S.V. Belyustin, Klassicheskaya electronnaya teoriya, Vysshaya shkola, Moskva, 1971 (Rus.)

[11] T. Katayama, T. Sugimoto, Y. Suzuki, M. Hashimoto, P. de Haan, J.C. Lodder, Magneto-optical Kerr rotation spectra in ordered and disordered phases of Fe-Pt alloys films, Journal of Magnetism and Magnetic Materials, 1992, Vol.104 - 107, $1002-1004$.

[12] Y.F. Ding, J.S. Chen, E. Liu, Structural and magnetic properties of nanostructured $\mathrm{FePt} / \mathrm{MgO}$ granular films, Thin Solid Films, 2005, Vol. 474, $141-145$.

[13] W.B. Mi, E.Y. Jiang, H.L. Bai, Particle size reduction and enchanced diffudion of $\mathrm{Fe}$ and $\mathrm{Pt}$ atoms in FePt-C granular films by $\mathrm{N}$ addition, Applied Surface Science, 2006, Vol. 253, $1830-1835$

[14] A. Asthana, Y.K. Takahashi, Yoshio Matsui, K.Hono, Effect of base pressure on the structure and magnetic properties of 
FePt thin films, Journal of Magnetism and Magnetic Materials, 2008, Vol. 320, $250-256$.

[15] A.S. Kamzin, E.V. Snetkova, Z. Yang, F. Wei, V. Hiang, Properties of thin FePt films synthesized by sequential sputtering of components, Technical Physics Letters, 2008,
Vol. 34, Iss.1, 26 - 29.

[16] C. Feng, B.H. Li, G. Han, J.Teng, Y. Jiang, T. Yang, G.H. Yu, Effect of the underlayer $(\mathrm{Ag}$, $\mathrm{Ti}$ or $\mathrm{Bi})$ on the magnetic properties of $\mathrm{Fe} / \mathrm{Pt}$ multilayer films, Thin Solid Films, 2007, Vol.515, 8009 - 8012 . 